\title{
Panel construction for mapping in admixed populations via expected mutual information
}

\author{
Sivan Bercovici, ${ }^{1,4}$ Dan Geiger, ${ }^{1}$ Liran Shlush, ${ }^{2}$ Karl Skorecki, ${ }^{2}$ and Alan Templeton ${ }^{3}$ \\ ${ }^{1}$ Computer Science Department, Technion, Haifa 32000, Israel; ${ }^{2}$ Faculty of Medicine, Technion, Haifa 31096, Israel; \\ ${ }^{3}$ Department of Biology, Washington University, St. Louis, Missouri 63130, USA
}

\begin{abstract}
Mapping by admixture linkage disequilibrium (MALD) is an economical and powerful approach for the identification of genomic regions harboring disease susceptibility genes in recently admixed populations. We develop an information-theory-based measure, called expected mutual information (EMI), which computes the impact of a set of markers on the ability to infer ancestry at each chromosomal location. We then present a simple and effective algorithm for the selection of panels that strives to maximize the EMI score. Finally, we demonstrate via well-established simulation tools that our panels provide more power and accuracy for inferring disease gene loci via the MALD method in comparison to previous methods.
\end{abstract}

Mapping by admixture linkage disequilibrium (MALD) is an economical and powerful approach for the identification of genomic regions harboring disease-susceptibility genes in recently admixed populations (Reich and Patterson 2005; Smith and O'Brien 2005). For the method to be useful, the prevalence of the disease under study should be considerably different between the ancestral populations from which the admixed population was formed.

Myeloma, for example, is a type of cancer that is approximately three times more prevalent in Africans than in Europeans (Smith and O'Brien 2005). Hepatitis C clearance is approximately five times more prevalent in Europeans than in Africans. Stroke, lung cancer, prostate cancer, dementia, end-stage renal disease, multiple sclerosis, hypertension, and many more diseases all exhibit a higher morbidity in either Africans or Europeans, when the two ethnically different populations are compared (Smith and O'Brien 2005). This difference in susceptibility to a specific disease is also evident in other ethnic populations. Native Americans suffer from a high prevalence of type 2 diabetes, obesity, and gallbladder disease, while showing a lower prevalence of asthma, relative to Europeans (Price et al. 2007).

When examining an individual who originated from several ancestral populations, such as African Americans, the likelihood that this individual will carry a given disease is influenced by the susceptibility to the disease in the ancestral populations. When such an admixed individual carries a hereditary disease, the chances are higher that the disease gene or genes are harbored in chromosomal segments that originated from the ancestral population with the higher risk.

The MALD method, also known as admixture mapping, screens through the genome of either affected or both affected and healthy admixed individuals, looking for chromosomal segments with an unusually high representation of the high-risk ancestral population for the disease. MALD requires 200-500fold fewer markers, in comparison to genome-wide association mapping, while offering the same power (Reich and Patterson 2005). Consequently, the method has an economical advantage over alternative methods. Lately, successful results from admix-

\section{${ }^{4}$ Corresponding author.}

E-mail sberco@cs.technion.ac.il; fax 972-4-8293900.

Article published online before print. Article and publication date are at http:// www.genome.org/cgi/doi/10.1101/gr.073148.107. ture mapping have begun to emerge. For example, the usage of MALD led to the discovery of multiple risk alleles (gene variants) for prostate cancer (Haiman et al. 2007).

In this study, we develop an information-theory-based measure, called expected mutual information (EMI), to select an effective panel of markers to be used in MALD. Our measure, presented below, computes the total impact of a set of markers on the ability to infer ancestry at each chromosomal location, averaged over all possible recombinations that could have occurred during the admixture process. This method improves previous measures such as the Shannon information content (SIC) (Rosenberg et al. 2003) and Fisher information content (FIC) (Pfaff et al. 2004). We then present a simple and effective algorithm for the selection of panels that strives to maximize the EMI score. Next, we demonstrate via well-established simulation tools used in previous studies that our panels provide more power for inferring disease gene loci. For example, when examining 576 cases from an admixed population comparative to the African-American population, our simulations show that in the challenging case of a disease with an ethnicity risk ratio of 1.6 between the two ancestral populations, assuming a multiplicative risk disease model, the power increased from $50 \%$ to $68 \%$, namely, an increase of $\sim 36 \%$ in the ability to detect the loci of diseasesusceptibility genes. The detection accuracy has also significantly improved with the use of our new panels. The increase in power is particularly important in the detection of weak signals that underlie complex diseases. We conclude with extensions and discussion.

\section{Background}

The MALD method consists of three steps. First, an admixed population with a significantly higher risk for a specific disease in one of the ancestral populations is identified. Ancestryinformative markers that effectively distinguish between the relevant ancestral populations are selected, and either case or both cases and controls are genotyped. Second, the ancestry along the chromosomes of every individual is computed based on the sampled genotypes. Third, chromosomal regions with an elevated frequency of the ancestral population with the higher disease prevalence are identified. Figure 1 illustrates the ancestral profile of eight individuals, of which half are cases and half con- 


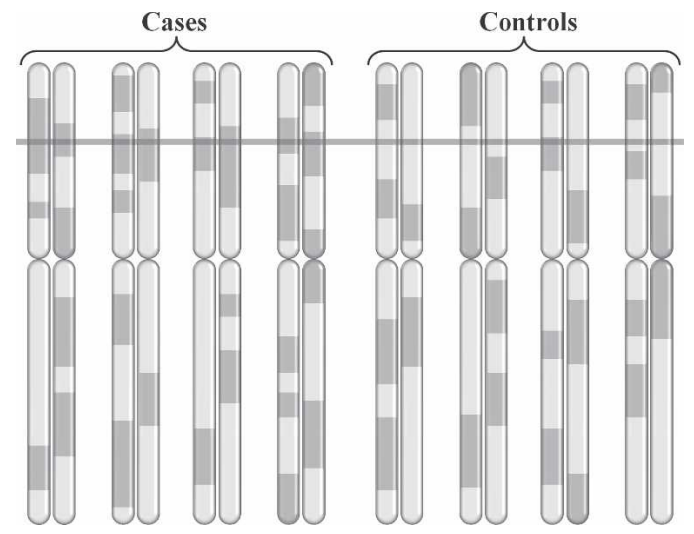

Figure 1. Ancestry informative markers are used to compute the ancestry across the chromosomes of cases and controls. The region indicated by the bar shows elevated frequency of the higher risk ancestral population in the cases versus the expected distribution of ancestry in the controls, suggesting a disease susceptibility locus.

trols. The ancestral profiles are indicated as dark and light segments along the chromosomes. The excess of the higher-risk ancestral population in the cases at the locus marked by the bar suggests that the locus contains the disease-susceptibility gene. In the controls, the ancestry at the same locus matches the expected distribution of ancestry under a given admixture model, strengthening the hypothesis that an association was found with a genuine disease locus. The detection of suspected regions can be followed by methods such as high-density SNP-based association studies, or a study of nearby candidate genomic regions.

Choosing ancestry-informative markers (AIM) for the construction of MALD panels has been pursued in several studies. AIM panels were constructed for African-American (Smith et al. 2004; Tian et al. 2006), Mexican-American (Tian et al. 2007), and Hispanic/Latino (Mao et al. 2007; Price et al. 2007) populations. The construction of such panels requires three ingredients: a database of markers, a measure for the informativeness of a set of markers regarding ancestry, and an algorithm that selects informative markers for the MALD panel.

The work of Rosenberg et al. (2003)) introduced a measurement for the information multialleleic markers provided on ancestry, based on the SIC. Pfaff et al. (2004) based their measurement on the FIC.

The algorithms used for panel construction in the studies that followed were driven by two prime objectives: (1) Choose markers with the highest ancestry-informativeness. (2) Choose evenly spread markers. These guidelines were set to provide informative panels for the estimation of ancestry at each point along the genome. Current panel construction algorithms are "greedy," attempting to locally maximize an informativeness criterion, while investing less effort in ensuring that the chosen markers are evenly spaced or that the informativeness along the genome is well balanced. Smith et al. (2004) used a purely greedy algorithm for marker selection. Tian et al. (2006) divided the chromosome into windows, choosing multiple highly informative markers within every such window.

When considering the informativeness of a set of markers regarding the ancestry at an arbitrary point, previous work offered rough approximations. Smith et al. (2004) considered the informativeness of a set of markers within a constant-size window centered on the point examined as an approximation to the informativeness at that point. Tian et al. (2006) used the mean informativeness between two adjacent markers bounding the point examined. It is this deficiency that is addressed in this study. In the next section, we develop an improved measure and demonstrate through simulations that panels constructed using our measure provide increased power in the detection of diseasesusceptibility gene loci.

\section{Admixed individuals model}

The genome of a recently admixed individual is a mosaic of large chromosomal segments, where each segment originated from a single ancestral population. We use the following definitions to describe these segments in admixed individuals.

Definition 1. An admixed chromosome is a chromosome that originated from more than one ancestral population.

Definition 2. A post-admixture recombination point (PAR) is a recombination point in which either two chromosomes from different populations crossed, or two chromosomes crossed when at least one of the chromosomes is an admixed chromosome.

Definition 3. A PAR block is a chromosomal segment limited by two consecutive PAR points, or by a chromosome edge and its closest PAR point.

An immediate implication of these definitions is that every PAR block originated from a single ancestral population, designated as the "ancestry of the block," for otherwise the block would have been further divided.

Figure 2 illustrates the propagation of PAR points along three generations of admixture, and the PAR blocks they induce. In particular, Figure 2 shows a grandmother originating from one population and a grandfather originating from two populations, yielding a parent with one admixed chromosome (with one PAR point) and one nonadmixed chromosome. As the parent's chromosomes recombine to produce the child's admixed chromosome, a second PAR point is added. Hence, three recombination points reside on the child's chromosome, of which only two are PAR points (colored black). Three PAR blocks are defined rather than four as the leftmost recombination point is not a PAR point.

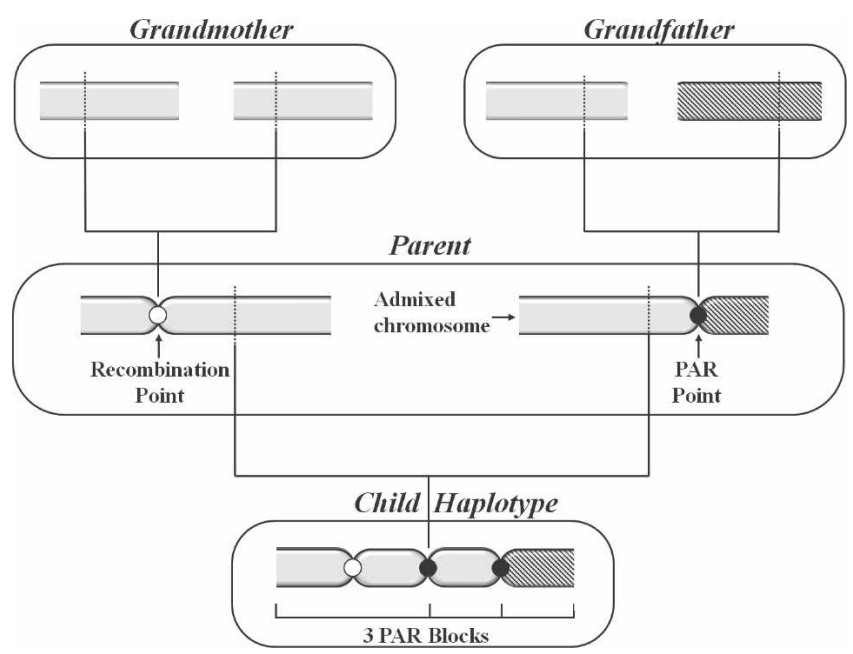

Figure 2. Three generations admixture example. PAR blocks are limited only by PAR points and the chromosomes' ends.

\section{Genome Research}

www.genome.org 
We denote the set of all observed markers by $J$, and the vector of an individual's PAR-blocks ancestries as $Q$. The set of an individual's PAR points defines a partition (denoted $\pi$ ) of the chromosomes into blocks. We use the random variable $Q_{\pi}$ to denote the vector of ancestries corresponding to the PAR blocks determined by $\pi, Q_{\pi, i}$ to denote the ancestry (out of $K$ possible ancestral populations) of the ith PAR block in the given partition $\pi$, and the random vector $J_{\pi, i}=\left\{J_{\pi, i, 1}, J_{\pi, i, 2}, \ldots, J_{\pi, i, m_{i}}\right\}$ to denote the set of $m_{\pi, i}$ observed markers within this block. Reference to subscript $\pi$ will be omitted whenever $\pi$ is clear from the context.

Markers within a PAR block are assigned according to the probability function of the corresponding ancestral population. We further assume that the ancestries of all PAR blocks of a given partition $\pi$ are mutually independent. A graphical model showing these assumptions is given in Figure 3.

The joint probability distribution described via the graphical model is given by

$$
P(Q, J)=\sum_{\pi} P(\pi) \cdot \prod_{i=1}^{\left|Q_{\pi}\right|} P\left(Q_{\pi, i}\right) \prod_{j=1}^{m_{\pi, i}} P\left(J_{\pi, i, j} \mid Q_{\pi, i}\right) .
$$

In particular, when considering a specific point $x$ on the genome, the joint probability for the ancestry $Q_{x}$ at that point is given by

$$
P\left(Q_{x}, J\right)=\sum_{\pi} P(\pi) \cdot P\left(Q_{x}, J_{\pi, x}\right) \cdot P\left(\bar{J}_{\pi, x}\right)
$$

where $J_{\pi, x}$ are the markers within the same PAR block as location $x$, and $\bar{J}_{\pi, x}$ is the complementary set of markers outside this block. We use this joint distribution to derive our panel informativeness measure.

\section{Informativeness of panels}

In this section, we develop a measure for the contribution of a set of observed markers to the ability to infer the ancestry of a block conditioned on a partition $\pi$. We then extend this measure to account for the fact that $\pi$ is unknown by computing the expectation over all possible partitions, while focusing on the inference of a single location $x$.

We start by exploring the relationship between observed markers and the ancestries of PAR blocks under the assumption that the partition is known. Using information theory, we estimate the extent to which a set of markers contribute to the ability to infer ancestry by measuring the informativeness of a set of

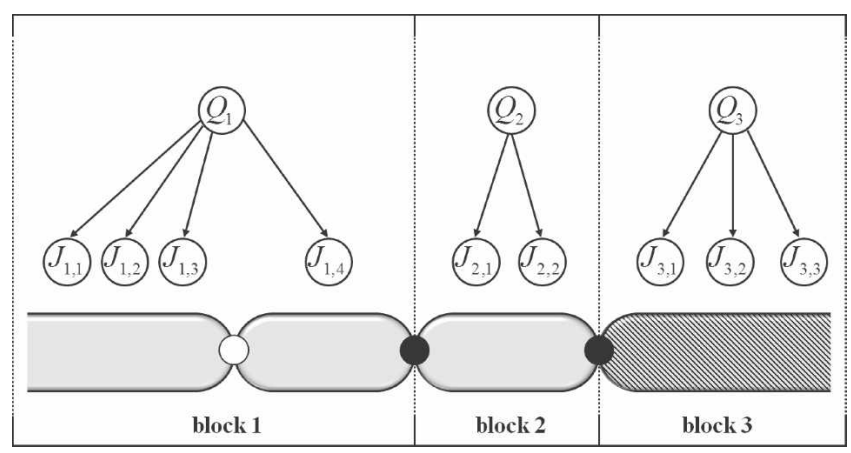

Figure 3. Graphical model for $P(Q, f)$ assuming markers $\int_{i}$ within a PAR block are independent conditioned on ancestry $Q_{i}$. Ancestries of PAR blocks are assumed to be mutually independent. markers regarding ancestry. The information gain for ancestry due to observing a set of markers can be described by the wellknown SIC:

$$
\begin{aligned}
I\left(Q_{i} ; J_{i}\right) & =H\left(Q_{i}\right)-H\left(Q_{i} \mid J_{i}\right) \\
& =\sum_{Q_{i}} \sum_{J_{i}} P\left(J_{i} \mid Q_{i}\right) \cdot P\left(Q_{i}\right) \cdot \log \frac{P\left(J_{i} \mid Q_{i}\right)}{P\left(J_{i}\right)},
\end{aligned}
$$

where $H\left(Q_{i}\right)$ is the entropy (or the amount of uncertainty) of the PAR block's ancestry, given by

$$
H\left(Q_{i}\right)=-\sum_{Q_{i}=1}^{K} P\left(Q_{i}\right) \cdot \log P\left(Q_{i}\right),
$$

and $H\left(Q_{i} \mid J_{i}\right)$ is the conditional entropy on ancestry once the markers observations are accounted for, given by

$$
H\left(Q_{i} \mid J_{i}\right)=-\sum_{Q_{i}} \sum_{J_{i}} P\left(J_{i}, Q_{i}\right) \cdot \log P\left(Q_{i} \mid J_{i}\right)
$$

In other words, the markers' informativeness is measured by the reduction in uncertainty regarding the ancestry of a given location due to observing these markers. This reduction in uncertainty originates from the fact that each ancestral population has a distinct distribution over the haplotype. The information gain in each PAR block is computed separately through Equation 3 due to our assumption of mutual independence.

The possible presence of linkage disequilibrium between markers within a block raises difficulties partially stemming from the need to estimate the joint probability of a haplotype $J_{i}$ that contains multiple markers conditioned on the ancestry [i.e., $\left.P\left(J_{i} \mid Q_{i}\right)\right]$. To reduce computational cost, we assume conditional independence between all markers given ancestry, yielding a simpler form of mutual information $I_{\text {ind }}\left(Q_{i} ; J_{i}\right)$, explicated in Lemma 1. The relaxation of this assumption is pursued in Section 7.

Lemma 1. For a given PAR block, let $Q_{i}$ be its ancestry, and $J_{i, j}$ be its jth marker (out of $m_{i}$ markers). Under the assumption that the markers are conditionally independent given $Q_{i}$, the mutual information between $Q_{i}$ and $J_{i}$ is:

$$
I_{\text {ind }}\left(Q_{i} ; J_{i}\right)=H\left(J_{i}\right)-\sum_{j=1}^{m_{i}} H\left(J_{i, j} \mid Q_{i}\right) .
$$

Given a partition $\pi$, all PAR blocks are determined, and the informativeness of markers regarding ancestry $Q_{i}$, and in particular regarding ancestry $Q_{x}$ of an arbitrary location $x$ within the $i$ th PAR block, is the informativeness of the markers in $J_{i}$ alone. All other markers, namely, $J \backslash J_{i}$, are not informative regarding $Q_{x}$. However, $\pi$ is not known, and for every $\pi$ a different block may contain location $x$, determining the set of markers that are informative regarding the ancestry at $x$. The expected informativeness of all markers regarding ancestry at location $x$ is given, in principle, by

$$
\operatorname{EMI}\left(Q_{x} ; J\right)=\sum_{\pi} P(\pi) \cdot I\left(Q_{x} ; J \mid \pi\right)
$$

We call this measure "EMI" for "expected mutual information." Since summing over all possible partitions is not feasible, the rest of this section rewrites Equation 5 and explicates how to compute it.

Observe that for any two partitions $\pi_{1}$ and $\pi_{2}$ such that the PAR block that contains location $x$ also contains the same set of 
markers $J_{\pi, x} \subseteq J$, the term $I\left(Q_{x} ; J \mid \pi\right)$ in Equation 5 is equal. The probability for a partition $\pi$ to contain a block that contains both location $x$ and markers $J_{\pi, x}$ is defined by three events:

1. The minimal segment $[l, r]$ that spans over $J_{\pi, x}$ and $x$ does not contain a PAR point.

2. The segment between $l$ and the marker to its left (at $l^{\prime}$ ), if such exists, contains a PAR point.

3. The segment between $r$ and the marker to its right (at $\left.r^{\prime}\right)$, if such exists, contains a PAR point.

Assuming that PAR points are distributed independently, the aforementioned three events are independent as well. This holds because the corresponding three segments are mutually exclusive. Hence, the probability of a partition $\pi$ to contain a PAR block containing location $x$ and markers $J_{\pi, x}$ alone is given by the product

$$
P_{(l, r)}=P\left(N_{\left[l^{\prime}, l\right]} \neq 0\right) \cdot P\left(N_{[l, r]}=0\right) \cdot P\left(N_{\left[r, r^{\prime}\right]} \neq 0\right),
$$

where $N_{[a, b]}$ is a random variable of the number of PAR points in segment $[a, b]$, and $[l, r]$ is the minimal segment containing location $x$ and markers $J_{\pi, x}$.

The term $P\left(N_{\left[l^{\prime}, l\right]} \neq 0\right)$ depends on the existence of a marker at $l^{\prime}$, hence, the term will equal 1 in Equation 6 in case there is no marker to the left of $l$. Similarly, $P\left(N_{\left[r, r^{\prime}\right]} \neq 0\right)$ will equal 1 in Equation 6 if there is no marker to the right of $r$.

Let $J_{[l, r]}$ denote a sequence of markers within a segment $[l, r]$, and location $(j)$ denote the location of a marker $j \in J$. To compute EMI, we weight the potential contribution $I\left(Q_{x} ; J_{[l, r]}\right)$ by the probability of such a contribution, namely, the probability $P_{(l, r)}$ of a partition $\pi$ to contain location $x$ and markers $J_{[l, r]}$ within the same block.

Theorem 1. Let $Q_{x}$ be the ancestry at location $x$, and $J$ the set of observed markers. The expected mutual information between $Q_{x}$ and $J$ is

$$
\operatorname{EMI}\left(Q_{x} ; J\right)=\sum_{l \in L} \sum_{r \in R} P_{(l, r)} \cdot I\left(Q_{x} ; J_{[l, r]}\right),
$$

where

$$
\begin{aligned}
& L=\{\operatorname{location}(j) \leq x \mid j \in \mathcal{J}\} \cup\{x\}, \\
& R=\{\operatorname{location}(j) \geq x \mid j \in J\} \cup\{x\} .
\end{aligned}
$$

A common assumption is that recombination points occur as a Poisson process (Patterson et al. 2004), hence, the realization of the term $P_{(l, r)}$ in Equation 7 is via the Poisson distribution. In particular,

$$
P\left(N_{[a, b]}=0\right)=e^{-\lambda \cdot|b-a|},
$$

where $\lambda$ is the rate of PAR points in admixed individuals, as derived from the admixture model being used. Consequently,

$$
P_{(l, r)}=\left(1-e^{-\lambda \cdot\left|l-l^{\prime}\right|}\right) \cdot e^{-\lambda \cdot|r-l|} \cdot\left(1-e^{-\lambda \cdot\left|r^{\prime}-r\right|}\right) .
$$

Equation 7 defines the EMI at a specific location $x$. The average information gain regarding the entire chromosome is given by

$$
\operatorname{EMI}_{\text {avg }}(J)=\frac{1}{|N|} \cdot \sum_{x \in N} \operatorname{EMI}\left(Q_{x}, J\right)
$$

which measures the average EMI along the chromosome. The set $N$ consists of all locations $x$ on an evenly spaced grid with a specific resolution. For example, for chromosome 1, a set $N$ of 280 points means about one location per centimorgan (cM).

In the task of mapping disease genes in admixed populations using the MALD method, panels of high $\mathrm{EMI}_{\mathrm{avg}}$ are shown below to outperform previous panels.

\section{Panel construction}

We employ a greedy algorithm that constructs panels of markers for which the $\mathrm{EMI}_{\mathrm{avg}}$ is high. In principle, the algorithm iterates over the candidate markers, selecting the marker with the highest $\mathrm{EMI}_{\text {avg }}$ gain given the markers chosen so far. Namely, in each iteration, the algorithm chooses a marker $j$ that maximizes

$$
\operatorname{EMI}_{\text {avg }}(J \cup\{j\})-\mathrm{EMI}_{\text {avg }}(J),
$$

where $J$ is the set of markers selected so far.

The evaluation of $\mathrm{EMI}_{\mathrm{avg}}$ is a computationally intensive task that is repeated with every iteration, and for every candidate marker. To reduce execution time, for each examined candidate, we locally evaluate $\mathrm{EMI}_{\mathrm{avg}}$ on a set of points located in a segment of length $w$ centered on the candidate marker. Equation 11 evaluates the $\mathrm{EMI}_{\text {avg }}$ on a subset of points $w_{j} \subseteq N$ :

$$
\operatorname{EMI}_{\text {avg }}(J)=\frac{1}{\left|w_{j}\right|} \cdot \sum_{x \in w_{j}} \operatorname{EMI}\left(Q_{x}, J\right)
$$

where

$$
w_{j}=\left\{p \in N \mid \operatorname{location}(j)-\frac{w}{2} \leq p \leq \operatorname{location}(j)+\frac{w}{2}\right\},
$$

rather than on the entire chromosome. Once a marker $j$ is chosen, the $\mathrm{EMI}_{\text {avg }}$ gain in the next iteration is computed only for those markers that are within $w_{j}$, as the last chosen marker mostly affects their potential gain.

The most computationally dominant factor in EMI is the evaluation of $H(J)$ (Equation 4), as it is exponential in the number markers $|J|$. However, for a given PAR block, a small number of ancestry-informative markers suffice to nearly eliminate the uncertainty regarding its ancestry; the information gain regarding the ancestry of the PAR block saturates rapidly as the number of informative markers within the PAR block increases. Hence, limiting the number of markers used in the evaluation of Equation 4 yields an eligible approximation. In our implementation, we limited the number of markers in the evaluation of Equation 4 to a maximum of 17 markers, offering a plausible trade-off between performance and approximation accuracy.

\section{Evaluation}

In this section, we demonstrate the power of panels produced by our algorithm and EMI. We compare performance with the works of Smith et al. (2004) and Tian et al. (2006).

Similarly to the panels of Smith et al. (2004) and Tian et al. (2006), we constructed a panel for the African-American admixed population. The International HapMap Project (The International HapMap Project 2005) was used as the SNP allele frequencies source for the two ancestral populations, namely, the West African and European populations. HapMap has been shown to reflect these two distinct populations well (Conrad et al. 2006).

\section{Genome Research}

www.genome.org 


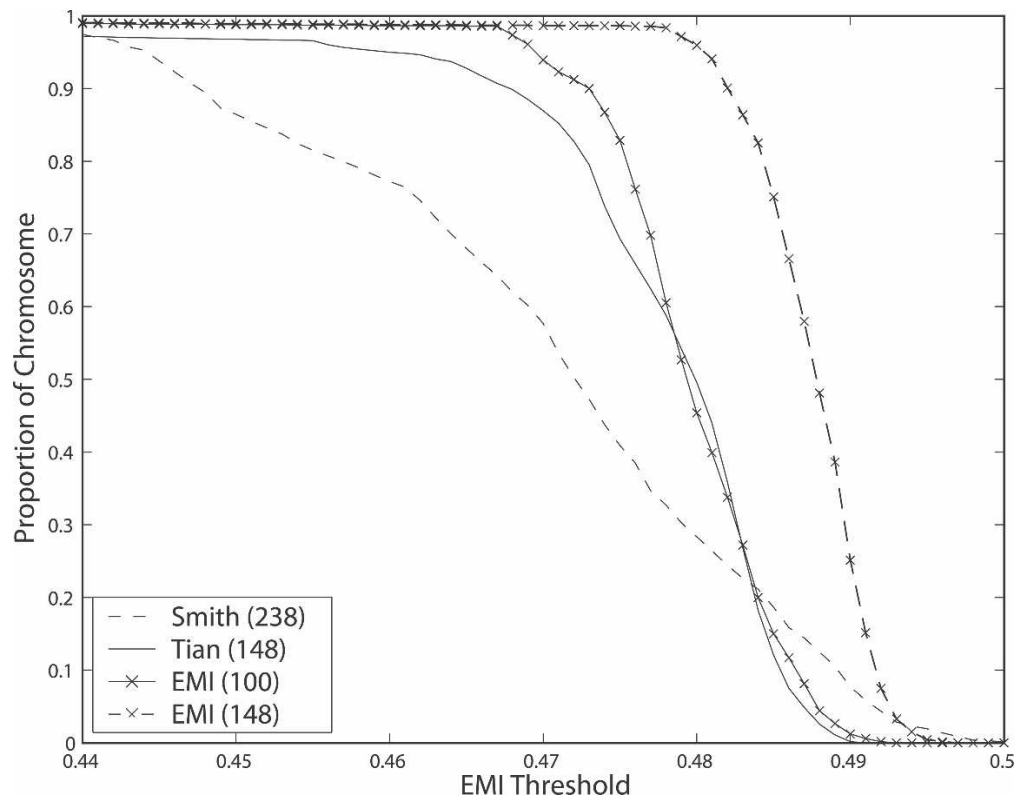

Figure 4. Proportion of chromosome above an EMI threshold. For most levels of informativeness, our panel covers larger segments of the chromosome.

We constructed a panel of 148 markers (denoted EMI-148) for chromosome 1, matching the number of corresponding markers in the screening panel of Tian et al. (2006) The panel of Smith et al. (2004) contains 238 markers. We further constructed a more economical panel of 100 markers for chromosome 1 (denoted EMI-100), which is two-thirds the number of markers in the panel constructed by Tian et al. (2006). Based on the admixture dynamics of African Americans as described by Hoggart et al. (2004), Smith et al. (2004), and Smith and O'Brien (2005), we used $\lambda=6$ (Equation 8) and a proportion of 0.8 African/0.2 European contribution to the admixed population. To compute $\mathrm{EMI}_{\mathrm{avg}}$ (Equation 11), $\mathrm{N}$ was defined as a set of evenly spaced locations along the chromosome at a resolution of $0.5 \mathrm{cM}$, yielding $N=574$ for chromosome 1 . We used a window size of $w=90$ $\mathrm{cM}$ because the expected informativeness of a marker decays to $\sim 5 \%$ for any location beyond $45 \mathrm{cM}$.

We first examine the performance of the four panels according to the EMI measure. The maximal EMI value is the entropy $H(Q)$. In the case of African Americans, the maximal EMI value is $\sim 0.5$ [namely, the entropy $H(Q)$ with $P\left(Q_{1}\right)=0.2$ and $P\left(Q_{2}\right)=0.8$ ]. Figure 4 illustrates that the informativeness of EMI-148 is considerably higher than previous panels. Moreover, the EMI-148 panel constructed by our algorithm has a low EMI standard deviation of 0.0041 in comparison to the screening panel of Tian et al. (2006) (0.0142) and the panel of Smith et al. (2004), (0.0178); indeed, our EMI measure strives to balance the informativeness of markers across the chromosome. It is interesting to note that our lighter panel, EMI-100, has good performance as well, with a low EMI standard deviation of 0.0056 .

ANCESTRYMAP (Patterson et al. 2004) is a tool we used for the estimation of the ancestral origin of a locus on the basis of sampled genotypes. Given genotypes of cases and controls, the tool can compute the likelihood of each point along the genome to be the disease locus. The disease model used by ANCESTRYMAP is a multiplicative risk model parametrized by the ethnicity relative risk (ERR) for the disease between the ancestral popula- tions (Patterson et al. 2004); having one allele from the higher-risk population inflicts a disease risk of ERR, while having two such alleles inflicts an $\mathrm{ERR}^{2}$ disease risk. ANCESTRYMAP can also generate samples of admixed-individual genotypes for cases and controls under this multiplicative disease model. This software was used in Smith et al. (2004) and Tian et al. (2006) to evaluate the power of the Smith and Tian panels, respectively.

In the experiments conducted, we generated 576 (some panel technologies use a sample size that is a multiplicative of 96) admixed-individual cases per experiment through the use of ANCESTRYMAP. In each experiment, a single location on chromosome 1 was used as the disease-predisposition locus. In order to evaluate the performance of the panel across the entire chromosome, a set of disease-predisposition loci was chosen using a resolution of four points per centimorgan; Consequently, 687 uniformly selected locations across chromosome 1 were used in the experiments. A range of ethnicity relative risk (ERR) factors, between 1.4 and 1.8, were set as the disease model parameter, all assuming that the European population exhibits the higher risk for the disease. We focused on this range as it captures diseases such as stroke and lung cancer (Smith and O'Brien 2005), which are considered mild in their ERR, hence harder to detect. We proceeded by employing ANCESTRYMAP to locate the disease gene. Similar to the threshold used for the evaluation of Tian's panel (Tian et al. 2006), we used a LOD score above 4.0 as an indicator for successful detection. Figure 5 shows the power, namely, the detection success rate, using 3435 experiments per panel.

Measuring the distance between the highest detected signal and the actual disease predisposition locus reveals that our panel

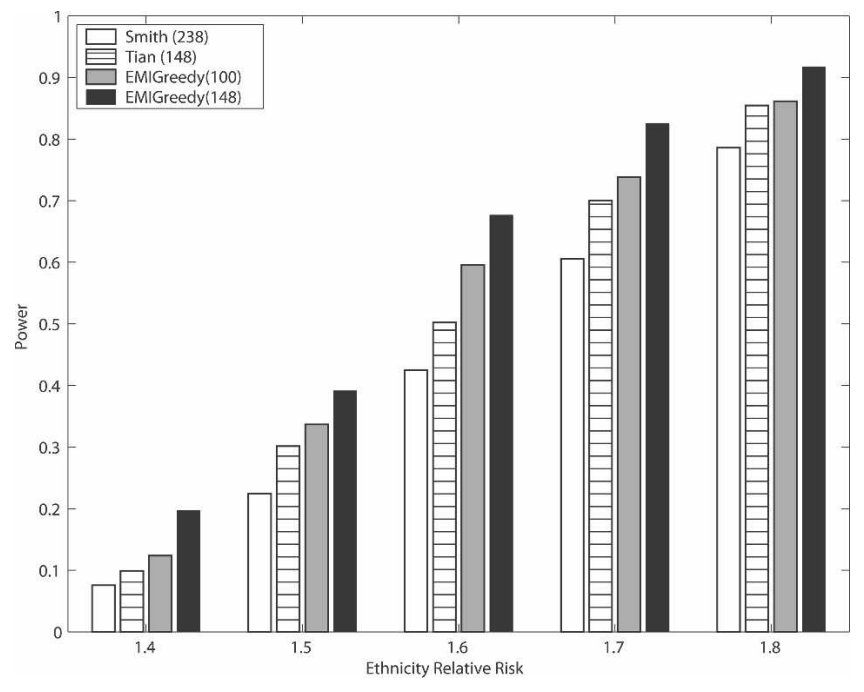

Figure 5. EMI-148 achieves a significantly higher power in all tested ERR values with 576 cases, assuming a multiplicative disease model. 
also has a higher detection accuracy, as Figure 6 illustrates for ERR 1.6.

Similar results hold for other ERR values within the tested range 1.4-1.8. Averaged over the range of ERR values tested, $\sim 55 \%$ of the experiments detected a signal within a 3-cM distance from the actual disease predisposition locus when EMI-148 was used, while the other two panels achieved $\sim 42 \%$ (Tian et al. 2006) and 37\% (Smith et al. 2004). The EMI-100 panel achieved an average of $46 \%$ detection rate within $3 \mathrm{cM}$ over the range of ERR values tested.

Observing the difference in performance between the EMI100 panel and the EMI-148 panel raises the question of power saturation, namely, under a given admixed population and a disease model, how many markers will suffice to reach near maximal power. We constructed five panels for chromosome 1 for African Americans, with a different number of markers ranging from 50 to 250 in steps of 50, and examined their power over a range of ERR values and 576 cases (200 experiments per configuration) using the multiplicative disease model. As illustrated in Figure 7 , for the case of an ERR of 1.7, the results indicate an increase in power from $46 \%$ (with 50 markers) to $84 \%$ (with 150 markers) and reaching $~ 89 \%$ (250 markers). Further increase in power requires significantly more markers, especially for $\mathrm{ERR} \geq 1.7$

We further tested the effect of sample size on the power of our panels. Figure 8 illustrates the power of EMI-148 over a range of ERR values with 200 experiments per configuration.

We compared the effect of sample size on power between the four panels using an ERR of 1.6. As illustrated in Figure 9, both EMI-148 and EMI-100 exhibit higher power under the different sample sizes tested, in comparison to the other two panels. More importantly, the results indicate that while the other two panels' power converged when more than 700 samples are used, our panels continued to benefit from the additional increase in sample size.

Detailed information regarding our panels is available at bioinfo.cs.technion.ac.il/MALD.

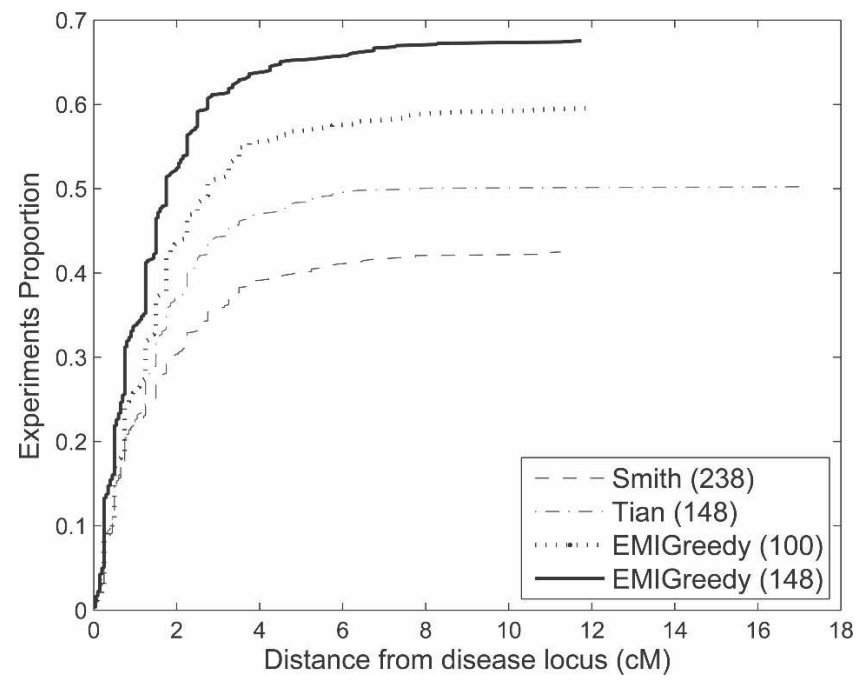

Figure 6. Experiments proportion up to an accuracy threshold for each of the four panels, under an ERR of 1.6 and 576 cases, assuming a multiplicative disease model. A higher proportion of the experiments yield higher accuracy for EMI-148, in comparison to the other three panels.

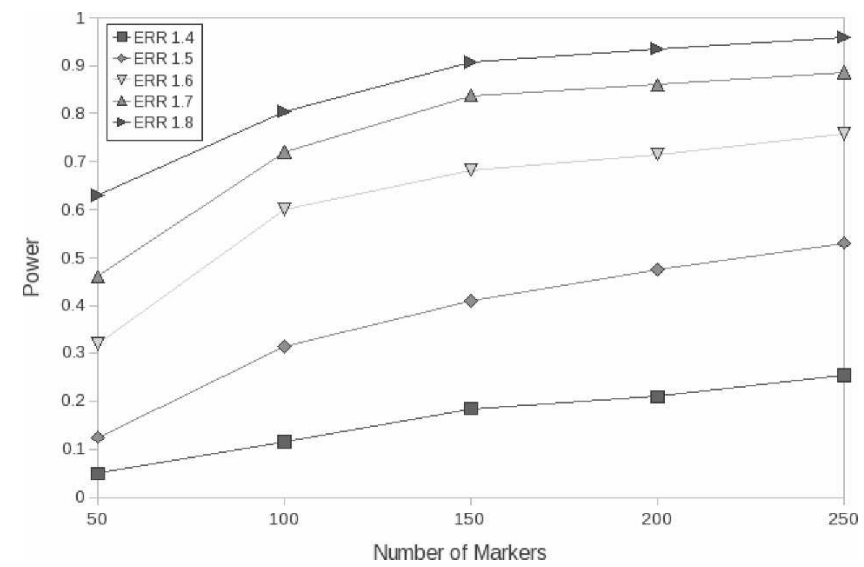

Figure 7. Power as a function of panel density, over a range of ERR values, using 576 cases and a multiplicative disease model.

\section{Extensions and Discussion}

The EMI measure provides an estimate for the informativeness of a set of markers regarding ancestry at a specific location. It improves upon previous measures as it takes into account the expected informativeness of a set of markers with respect to ancestry, over all possible partitions. The higher accuracy of EMI, especially in regions between markers, enables the creation of panels that are well balanced in terms of the informativeness provided by the set of markers across the genome. Finally, the panels constructed by our algorithm demonstrated significantly higher power and accuracy.

An immediate extension of EMI that we pursued addresses possible dependencies between markers given ancestry. Lemma 1 disregards LD within ancestral population in favor of a lower computational cost. We now use a first-order Markov-chain to model marker dependencies within ancestral populations in order to provide a more accurate model. Under this model, the transition probabilities are derived from the LD present between every two adjacent markers given the ancestry. Such a model still yields a computationally plausible form, as shown in the next lemma.

Lemma 2. For a given PAR Block, let $Q_{i}$ be the ancestry, and $J_{i, j}$ be the jth marker (out of $m_{i}$ markers). Under the assumption that each marker is dependent on the preceding marker and conditionally independent of the rest of the markers given $Q_{i}$, the mutual information of $Q_{i}$ and $J_{i}$ is:

$$
I_{\text {chain }}\left(J_{i} ; Q_{i}\right)=H\left(J_{i}\right)-H\left(J_{i, 1} \mid Q_{i}\right)-\sum_{j=2}^{m_{i}} H\left(J_{i, j} \mid Q_{i}, J_{i, j-1}\right) .
$$

Another extension of EMI relaxes the assumption that the rate of PAR points, used in Equation 8, is constant across the chromosomes. Recombinational hotspots can be taken into account by using a PAR point rate as a function of location $\lambda(x)$ instead of the constant rate $\lambda$. For example, assume that a chromosome is divided into regions of different PAR point rates $\lambda_{1}$, $\lambda_{2}, \ldots, \lambda_{n}$. For a segment $[l, r]$ that spans two consecutive regions with PAR rates $\lambda_{i}$ and $\lambda_{i}+1$, the term $P\left(N_{[l, r]}\right)$ in Equation 6 equals $e^{-\left(\lambda_{i} \cdot t+\lambda_{i+1} \cdot(1-t)\right) \cdot|r-l|}$, where $t$ is the proportion of segment $[l, r]$ with PAR rate $\lambda_{i}$.

Differences in the rate and distribution of recombination points in the ancestral population affect our assumption that the

\section{Genome Research}

www.genome.org 


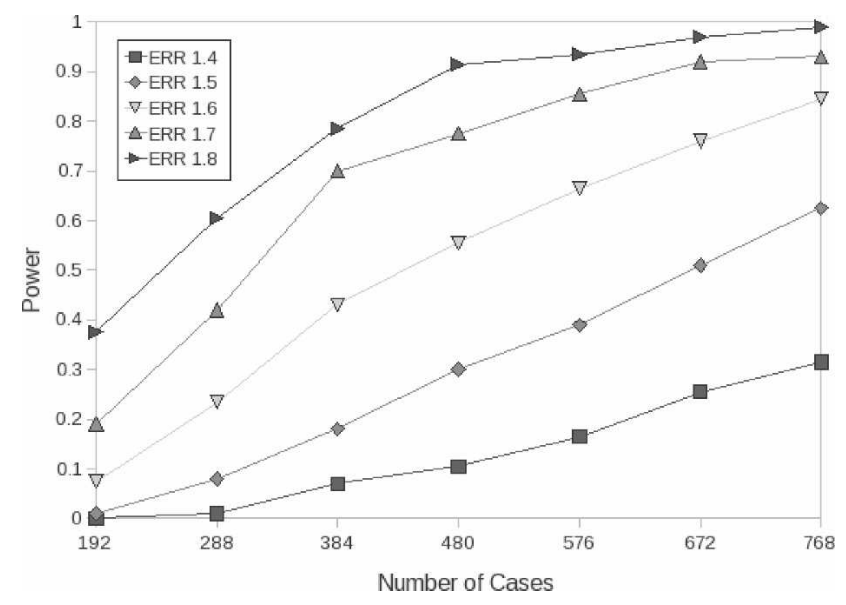

Figure 8. Power of EMI-148 as a function of the number of cases used for several ERR values assuming a multiplicative disease model.

ancestries of PAR blocks are mutually independent. Studying such differences and their extent, as was done in Conrad et al. (2006), will enable us to extend our measure accordingly.

We note that EMI assumes a model for haplotypes rather than genotypes, and that the allele frequencies $P(J \mid Q)$ are definite. In reality, these frequencies are derived from a small set of samples (60, barring missing data, in the case of HapMap). In its current form, EMI lacks an appropriate treatment for the uncertainty involving allele frequencies. It is advisable to validate the allele frequencies by taking more samples for candidate markers, as done in Tian et al. (2006).

The approach presented in this paper for panel construction also applies to the second phase of the MALD method. This phase currently employs a Markov chain model that assigns the most probable ancestry for each location, given the model and marker data (Hoggart et al. 2004; Patterson et al. 2004). By conditioning on possible partitions $\pi$, one can compute the expected ancestry $P\left(Q_{x} \mid J=j\right)$ at a point $x$ given measurements $J=j$ via Equation 2, similarly to our computation of the expected informativeness. It would be interesting to see whether this approach yields higher accuracy in ancestry inference.

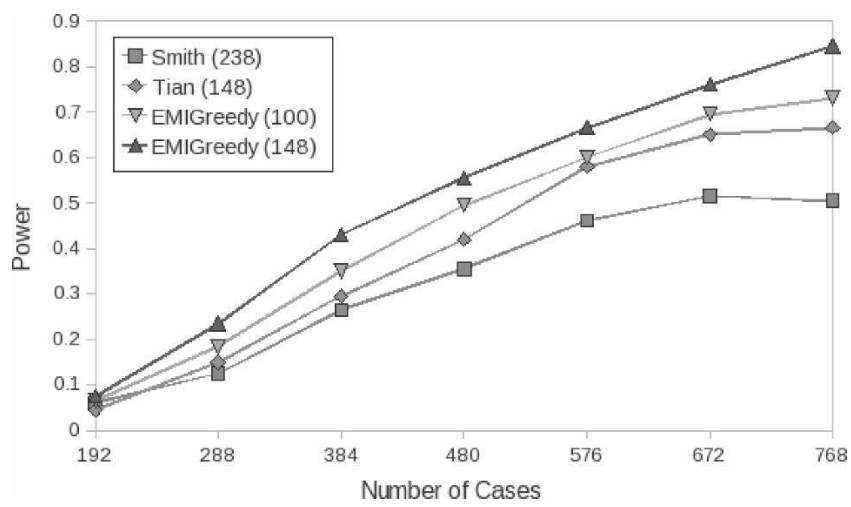

Figure 9. The power of the four panels as a function of the number of cases used for ERR 1.6 and a multiplicative disease model.
In summary, we showed that the panels produced using EMI have a well-balanced high score in terms of informativeness of markers, yielding a significant improvement in both power and accuracy, compared to previous work.

\section{Acknowledgments}

We thank Walter Wasser and Guennady Yudkovsky for fruitful discussions, Mark Silberstein for support, Tamar Aizikowitz for her helpful comments, and the reviewers for thoughtful comments. We also thank the Wolfson Foundation for contributing a grid of 120 PCs that enabled us to conduct this research. This research is supported by a Microsoft TCI grant.

\section{References}

Conrad, D.F., Jakobsson, M., Coop, G., Wen, X., Wall, J.D., Rosenberg, N.A., and Pritchard, J.K. 2006. A worldwide survey of haplotype variation and linkage disequilibrium in the human genome. Nat. Genet. 38: 1251-1260.

Haiman, C.A., Patterson, N., Freedman, M.L., Myers, S.R., Pike, M.C., Waliszewska, A., Neubauer, J., Tandon, A., Schirmer, C., McDonald, G.J., et al. 2007. Multiple regions within 8q24 independently affect risk for prostate cancer. Nat. Genet. 39: 638-644.

Hoggart, C.J., Shriver, M.D., Kittles, R.A., Clayton, D.G., and McKeigue, P.M. 2004. Design and analysis of admixture mapping studies. Am. J. Hum. Genet. 74: 965-978.

The International HapMap Project. 2005. A haplotype map of the human genome. Nature 437: 1299-1319.

Mao, X., Bigham, A., Mei, R., Gutierrez, G., Weiss, K., Brutsaert, T., Leon-Velarde, F., Moore, L., Vargas, E., McKeigue, P., et al. 2007. A genome-wide admixture mapping panel for Hispanic/Latino populations. Am. J. Hum. Genet. 80: 1171-1178.

Patterson, N., Hattangadi, N., Lane, B., Lohmueller, K.E., Hafler, D.A., Oksenberg, J.R., Hauser, S.L., Smith, M.W., O'Brien, S.J., Altshuler, D., et al. 2004. Methods for high-density admixture mapping of disease genes. Am. J. Hum. Genet. 74: 979-1000.

Pfaff, C.L., Barnholtz-Sloan, J., Wagner, J.K., and Long, J.C. 2004. Information on ancestry from genetic markers. Genet. Epidemiol. 26: $305-315$.

Price, A.L., Patterson, N., Yu, F., Cox, D.R., Waliszewska, A., McDonald, G.J., Tandon, A., Schirmer, C., Neubauer, J., Bedoya, G., et al. 2007. A genomewide admixture map for Latino populations. Am. J. Hum. Genet. 80: 1024-1036.

Reich, D. and Patterson, N. 2005. Will admixture mapping work to find disease genes? Philos. Trans. R. Soc. Lond. B Biol. Sci. 360: 1605-1607.

Rosenberg, N.A., Li, L.M., Ward, R., and Pritchard, J.K. 2003. Informativeness of genetic markers for inference of ancestry. Am. J. Hum. Genet. 73: 1402-1422.

Smith, M.W. and O'Brien, S.J. 2005. Mapping by admixture linkage disequilibrium: Advances, limitations and guidelines. Nat. Rev. Genet. 6: 623-632.

Smith, M.W., Patterson, N., Lautenberger, J.A., Truelove, A.L., McDonald, G.J., Waliszewska, A., Kessing, B.D., Malasky, M.J., Scafe, C., Le, E., et al. 2004. A high-density admixture map for disease gene discovery in African Americans. Am. J. Hum. Genet. 74: 1001-1013.

Tian, C., Hinds, D.A., Shigeta, R., Kittles, R., Ballinger, D.G., and Seldin, M.F. 2006. A genomewide single-nucleotidepolymorphism panel with high ancestry information for African American admixture mapping. Am. J. Hum. Genet. 79: 640-649.

Tian, C., Hinds, D.A., Shigeta, R., Adler, S.G., Lee, A., Pahl, M.V., Silva, G., Belmont, J.W., Hanson, R.L., Knowler, W.C., et al. 2007. A genomewide single nucleotide polymorphism panel for Mexican American admixture mapping. Am. J. Hum. Genet. 80: 1014-1023.

Received October 17, 2007; accepted in revised form February 12, 2008. 


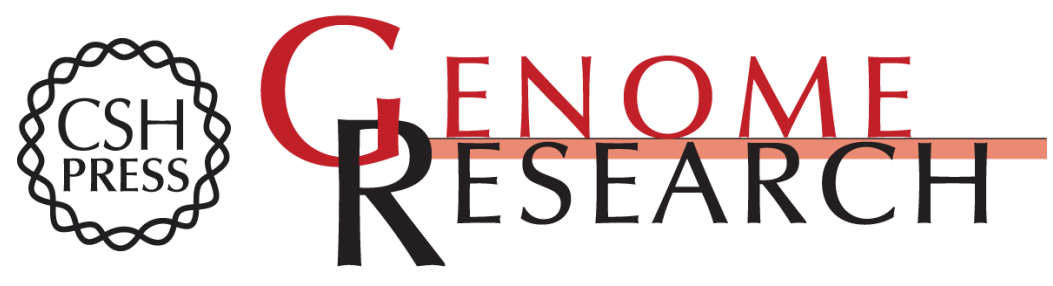

\section{Panel construction for mapping in admixed populations via expected mutual information}

Sivan Bercovici, Dan Geiger, Liran Shlush, et al.

Genome Res. 2008 18: 661-667 originally published online March 18, 2008

Access the most recent version at doi:10.1101/gr.073148.107

\section{Related Content Increasing power in association studies by using linkage disequilibrium structure and molecular function as prior information \\ Eleazar Eskin \\ Genome Res. April , 2008 18: 653-660 On the inference of ancestries in admixed populations \\ Sriram Sankararaman, Gad Kimmel, Eran Halperin, et al. \\ Genome Res. April , 2008 18: 668-675 Effect of genetic divergence in identifying ancestral origin using HAPAA \\ Andreas Sundquist, Eugene Fratkin, Chuong B. Do, et al. \\ Genome Res. April , 2008 18: 676-682}

\section{License}

Email Alerting

Receive free email alerts when new articles cite this article - sign up in the box at the Service top right corner of the article or click here.

\section{Affordable, Accurate Sequencing.}

